\section{In vivo trans-rectal ultrasound-coupled optical tomography of a transmissible venereal tumor model in the canine pelvic canal}

\author{
Zhen Jiang, ${ }^{\text {a }}$ G. Reed Holyoak, ${ }^{\text {b Kenneth E. Bartels, }}$ \\ Jerry W. Ritchey, ${ }^{\mathrm{c}}$ Guan Xu, ${ }^{\mathrm{a}}$ Charles F. Bunting, ${ }^{\mathrm{a}}$ \\ Gennady Slobodov, ${ }^{\mathrm{d}}$ and Daqing Piao ${ }^{\mathrm{a}, *}$ \\ ${ }^{a}$ Oklahoma State University, School of Electrical and \\ Computer Engineering, Stillwater, Oklahoma 74078 \\ ${ }^{\mathrm{b}}$ Oklahoma State University, Department of Veterinary \\ Clinical Sciences, Stillwater, Oklahoma 74078 \\ 'Oklahoma State University, Department of Veterinary \\ Pathobiology, Stillwater, Oklahoma 74078 \\ ${ }^{\mathrm{d} U n i v e r s i t y ~ o f ~ O k l a h o m a ~ H e a l t h ~ S c i e n c e s ~ C e n t e r, ~}$ \\ Department of Urology, Oklahoma City, Oklahoma 73104
}

\begin{abstract}
In vivo trans-rectal near-infrared (NIR) optical tomography was performed concurrently with, albeit reconstructed without spatial a prior of, trans-rectal ultrasound (US) on transmissible venereal tumor (TVT) developed as a model in the canine pelvic canal. Studies were taken longitudinally at prior to, 14 days after, and 35 days after the TVT injection. As the tumor grew, the nodules became increasingly hyperabsorptive and moderately hyperscattering on NIR. The regions of strong NIR contrast, especially on absorption images, correlated well with those of US hypoechoic masses indicative of tumors. Combining the information of trans-rectal NIR and US detected the tumor more accurately than did the US alone at 14 days postinjection. $\odot 2009$ Society of Photo-Optical Instrumentation Engineers. [DOl: 10.1117/1.3149852]
\end{abstract}

Keywords: prostate cancer; trans-rectal optical tomography; transmissible venereal tumor; trans-rectal ultrasound

Paper 09010LR received Jan. 14, 2009; revised manuscript received Mar. 9, 2009; accepted for publication Apr. 17, 2009; published online Jun. 8, 2009.

\section{Introduction}

Near-infrared (NIR) optical tomography is becoming increasingly important for functional imaging of biological tissues ${ }^{1}$ because the endogenous or exogenous NIR contrast could benchmark tissue physiology and functionality. NIR optical tomography has contributed to diagnosis and prognosis of cancer, ${ }^{2}$ understanding of cerebral response ${ }^{3}$ characterization of rheumatologic dysfunction, ${ }^{4}$ and small animal imaging. ${ }^{5}$ In all these applications, the tissues have been interrogated noninvasively via external NIR applicators.

NIR optical tomography based on trans-rectal "noninvasive" probing has been investigated recently by the authors, attempting to augment trans-rectal ultrasound (TRUS) ${ }^{6}$ of

*Address all correspondence to: Daqing Piao, Tel: 405-744-5250; E-mail: daqing.piao@okstate.edu prostate with the unique optical specificity. This approach was motivated by the hypothesis that optical properties of prostate cancer in vivo may be different from those of normal intact prostate tissues and was challenged by the difficulty of assessing the prostate in its in vivo real-time environment. This work demonstrates in vivo trans-rectal NIR optical tomography, conducted concurrently with TRUS, on a dog bearing transmissible venereal tumors (TVTs) ${ }^{7}$ in its pelvic canal. This study not only validates the utility of in vivo trans-rectal NIR tomography of the prostate but also reveals, for the first time, the NIR contrasts that TVT has over the normal tissues within the canine pelvic canal.

\section{Methods and Materials}

The studies were conducted under a protocol approved by the Institutional Animal Care and Use Committee of Oklahoma State University. The protocol was also approved and underwent an on-site inspection by the U.S. Army Medical Research and Material Command. For this study, the prostate of a 12-kg sexually intact adult purpose-bred Beagle dog estimated to be approximately four years of age was used. The TVT cell line was obtained cryopreserved from MD Anderson Cancer Center (Houston, Texas). Following two cycles of inoculation into the subcutis of non-obese-diabetic/severecombined-immunodeficiency (NOD/SCID) mice, neoplastic cells were recovered and homogenized for injection into the canine prostate gland. Approximately $3 \mathrm{cc}$ of TVT cells were aseptically injected transperineally into the right lobe of the prostate using a 6-in. 16-gauge hypodermic needle under TRUS visualization. During retraction of the injection needle, it was assumed that TVT cells could leak from the prostate injection site and be "seeded" along the needle insertion tract. During the first 14 days postinjection, there was no evidence of tumor growth on TRUS and rectal examination. The TRUS examination at 35 days postinjection showed hypoechoic masses in the prostatic parenchyma, periprostatically around the right lobe of the prostate, and perirectaly along the track of needle injection. The dog underwent weekly monitoring for two more weeks and was then humanly euthanized for necropsy and histological examinations at 56 days postinjection.

The trans-rectal NIR/US system was described elsewhere. ${ }^{6}$ The NIR probe has been integrated with a 60 -mm-long sagittal TRUS transducer. The $60-\mathrm{mm}$ length of the NIR source/ detector arrays limits the imaging depth to $\sim 30 \mathrm{~mm}$. The steady-state NIR measurements reconstruct the absorption and reduced scattering images without a priori structural information. ${ }^{6}$

\section{Results}

Figure 1 presents the trans-rectal NIR and US images acquired at the middle of the right lobe, the middle line of the prostate, and the middle of the left lobe, which were obtained before the TVT injection, 14 days postinjection, and 35 days postinjection. The absorption [Fig. 1(a)] and reduced scattering [Fig. 1(b)] images correlate to the same set of US images. The image dimensions are $60 \times 30 \mathrm{~mm}^{2}$ (cranial-caudal $\times$ dorsal-ventral). On the day-35 US images of the right lobe

1083-3668/2009/14(3)/030506/3/\$25.00 @ 2009 SPIE 


\section{JBO LETTERS}

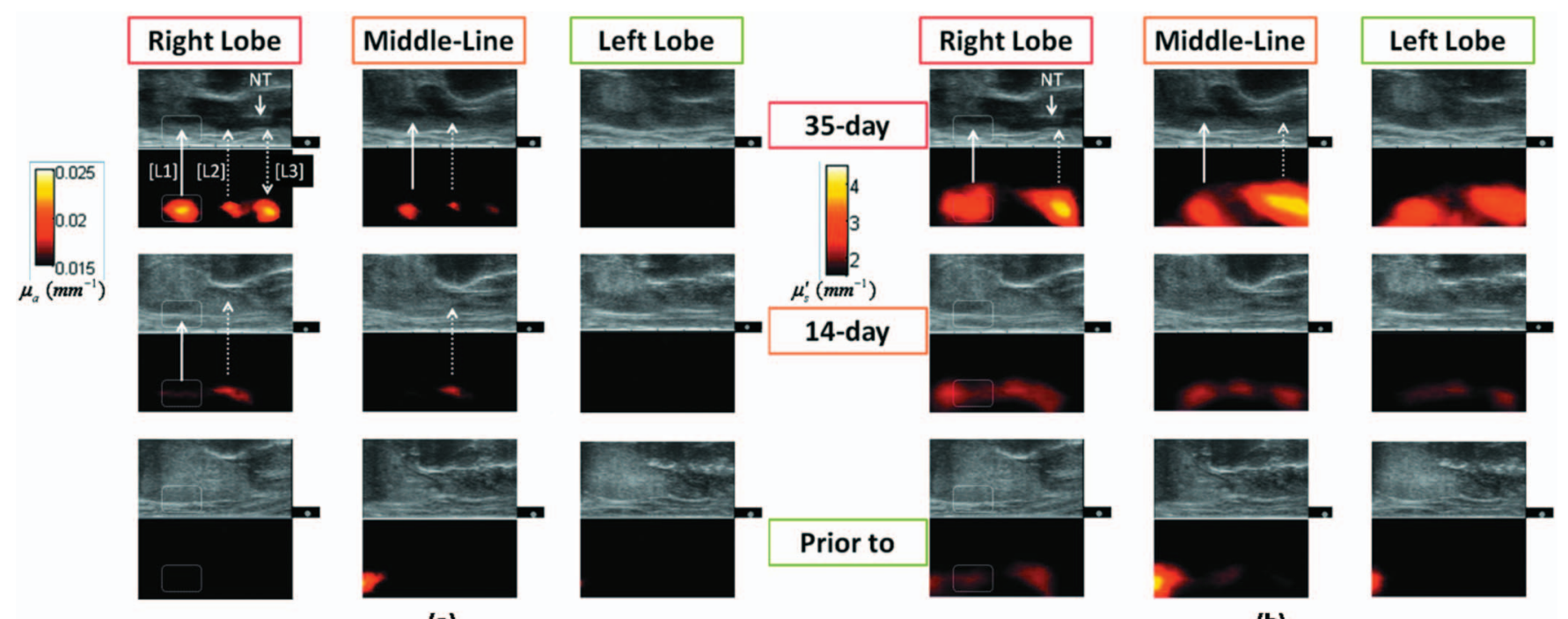

(a)

(b)

Fig. 1 In vivo trans-rectal NIR/US of TVT development in canine pelvic canal. The images were taken before the TVT injection, 14 days after the TVT injection when the US and rectal examination showed no evidence of tumor growth, and 35 days after the TVT injection when the tumor growth was evident on both US and rectal examination. (a) US and NIR absorption images. (b) US and NIR reduced scattering images. The dimensions of the images are $60 \times 30 \mathrm{~mm}^{2}$ (cranial-caudal $\times$ dorsal-ventral). The rectangular mark enclosing L1 is used in Fig. 2(a), where the peak NIR contrasts within the region are plotted. NT: needle track.

and middle line, the hypoechoic region "L1" indicated an intraprostatic mass; the large hypoechoic region "L2" indicated a mass ventral and caudal to the prostate that could have a connection with L1; the needle track (NT) on the right lobe denoted the needle trajectory for introducing the TVT cells with longitudinal hypoechoic regions, including L3, seen along the NT. On the day-35 NIR image of the right lobe, the hyperabsorptive regions corresponded longitudinally to L1, L2, and L3. The day-35 NIR image of the middle line displayed less and smaller hyperabsorptive masses, indicative of L1, L2, and L3. Trans-rectal NIR/US images performed at the left lobe were shown with no abnormal features on US and no hyperabsorptive regions on NIR. At the day-14 NIR absorption images, hyperabsorptive regions were found intraprostatically in the right lobe only (L1) and dorsal to the pelvic bone in the right lobe (L2), with potential extension to the middle line. The longitudinal locations of these hyperabsorptive regions correlated well with those of the hypoechoic and hyper- absorptive regions found in day-35. Because the NIR array surface is $3 \mathrm{~mm}$ ventral to the TRUS surface, the nodules were shown to be slightly dorsal on the NIR versus on the US images. The change of the hyperabsorptive regions from day-14 to day-35 implied tumor growing in the right lobe and extending toward the middle line. The growth of the tumor was indicated earlier by the NIR absorption images than by the TRUS, and combining the information of NIR and TRUS led to earlier and more accurate findings of tumor growth than did TRUS alone.

The hyperabsorptive masses in Fig. 1(a) were shown in Fig. 1(b) with different patterns of the contrast. In Fig. 1(b), the L3 had much higher contrast than did the other masses corresponding to L1 and L2. The growth of tumors L1 and L2 at day-14 in Fig. 1(b) are not as evident as in Fig. 1(a).

The progressions of the peak NIR contrasts within the rectangular region corresponding to L1 as outlined in Fig. 1, with

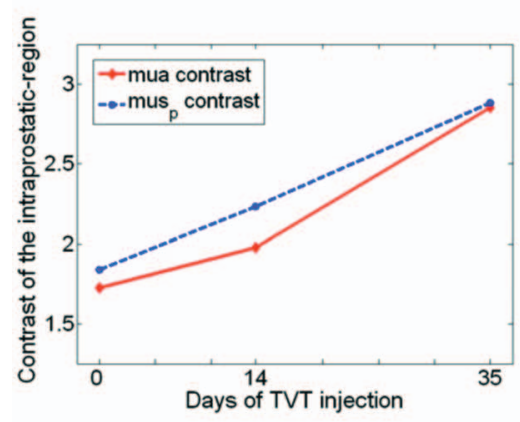

(a)

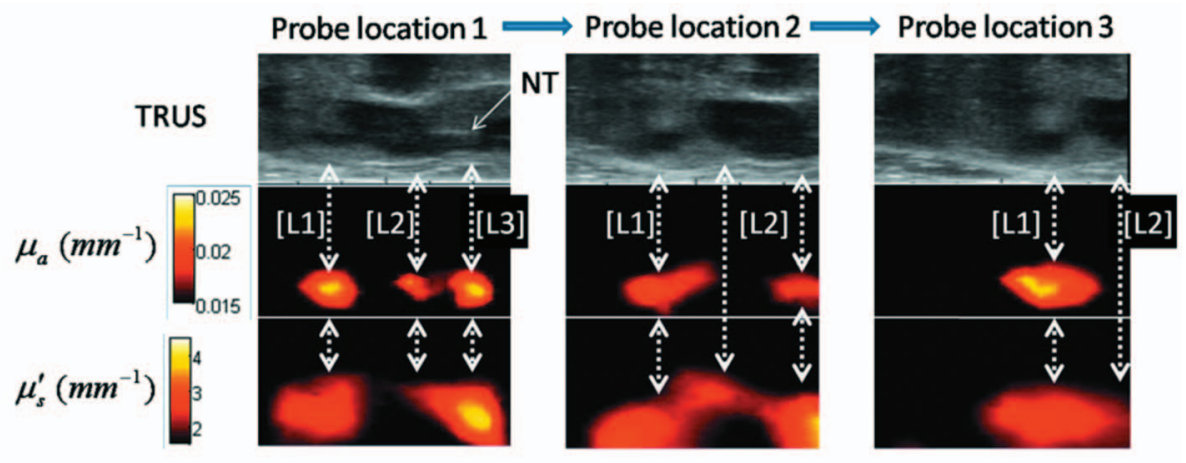

(b)

Fig. 2 (a) The progression of peak NIR contrasts within the rectangular region specified in Fig. 1. (b) Sagittal trans-rectal NIR/US imaging in three longitudinal locations. The locations of the left-column images correspond to those of the right lobe taken at day-35 in Fig. 1. 


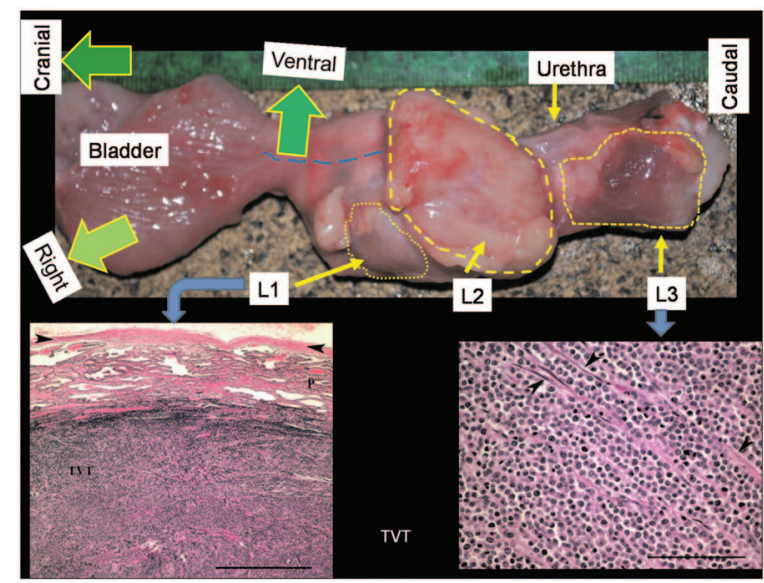

Fig. 3 (Top) Image of prostate gland taken after removal of the urinary bladder and intrapelvic urethra immediately following euthanasia. The prostate gland is markedly expanded and misshapen secondary to intraprostatic (L1) and periprostatic (L2) neoplastic masses. Neoplastic masses were also distributed along the distal intrapelvic urethra (L3) and in the perirectal subcutaneous tissues (not shown). Histologically, expansile masses of TVT (cells bottom left panel) displace and compress prostatic tissue (P). Prostatic capsule (arrowheads), bar $=1.8 \mathrm{~mm}$. The TVT infiltrate (bottom right panel) consists of sheets of neoplastic round cells dissecting through preexisting fibrovascular stroma (arrowheads), bar $=230 \mu \mathrm{m}$.

respect to the background values for normal perirectal parenchyma, are given in Fig. 2(a). The clear trend of contrast increase is believed as the demonstration of imaging the tumor growth because NIR tomography is a nonlinear process in which the enlargement of the target may be reconstructed as having elevated contrast in addition to volume changes when reconstructed without the spatial prior information of the target.

Figure 2(b) displays the NIR/US images obtained by placing the probe at three locations along the middle plane of the right lobe. At location 1, L1, L2, and L3 (refer to Fig. 1) were all within the US field of view, whereas at location 2 the L3 was moved out of the US view. At location 3, only L1 was shown on US. The overall NIR hypercontrast masses correlated well with the US hypoechoic regions.

The gross and histological findings (56 days postinjection) in Fig. 3 confirmed intra- and periprostatic neoplastic infiltrates with masses also located along the urethra and perirectal tissue; the latter related to dissemination along the needle track during TVT inoculation. All masses consist of diffuse sheets of a monomorphic population of neoplastic round cells dissecting through preexisting fibrovascular stroma. The neoplastic cells have large hyperchromatic nuclei, single conspicuous nucleoli, and moderate amounts of featureless cytoplasm. The cytological features are consistent with canine TVT.

\section{Discussions and Conclusion}

The intraprostatic TVT tumors were initiated in a nonimmunosuppressed canine model in which the TVT nodules developed at multiple sites intraprostatically and periprostatically. Although not all TVT tumors were confined to the prostate, successful imaging of multiple TVT nodules implies the utility of detecting multiple intraprostatic tumors.

The feature of TVT as strongly hyperabsorptive on NIR tomography is likely due to the hyperchromatic nuclei unique to TVT. The relatively higher scattering of the TVT may be due to the hyperdensity and larger nuclei of the neoplastic cells. As the neoplastic cells are arranged into microlobules by the preexisting fibrovascular stroma, the TVT may also present certain polarization sensitivity. Overall, the NIR features of TVT may be comparable to those revealed by tissue angiogenesis. As studies of microvessel-density within the human prostate demonstrated a clear correlation of increased microvessel density with the presence of cancer, ${ }^{8}$ it can be expected that human prostate cancer may have notable contrast on trans-rectal NIR tomography.

In conclusion, this work reports in vivo imaging of TVT tumors in the canine pelvic canal by trans-rectal NIR tomography coupled with TRUS. The TVT tumor nodules were presented as hyperabsorptive and hyperscattering with respect to the normal prostatic and other pelvic tissues. Correlation of the TVT locations is found between trans-rectal NIR and TRUS images. These demonstrations encourage testing of trans-rectal NIR tomography with additional animal studies and eventually to the human prostate.

\section{Acknowledgments}

The authors acknowledge support from U.S. Army Medical Research and Material Command through Grant No. W81XWH-07-1-0247.

\section{References}

1. E. M. Hillman, "Optical brain imaging in vivo: techniques and applications from animal to man," J. Biomed. Opt. 12, 051402 (2007).

2. B. J. Tromberg, B. W. Pogue, K. D. Paulsen, A. G. Yodh, D. A. Boas, and A. E. Cerussi, "Assessing the future of diffuse optical imaging technologies for breast cancer management," Med. Phys. 35(6), 2443-2451 (2008).

3. B. W. Zeff, B. R. White, H. Dehghani, B. L. Schlaggar, and J. P. Culver, "Retinotopic mapping of adult human visual cortex with high-density diffuse optical tomography," Proc. Natl. Acad. Sci. U.S.A. 104, 12169-12174 (2007).

4. A. K. Scheel, M. Backhaus, A. D. Klose, B. Moa-Anderson, U. J. Netz, K. G. Hermann, J. Beuthan, G. A. Müller, G. R. Burmester, and A. H. Hielscher, "First clinical evaluation of sagittal laser optical tomography for detection of synovitis in arthritic finger joints," Ann. Rheum. Dis. 64, 239-245 (2005).

5. M. B. Unlu, Y. Lin, O. Birgul, O. Nalcioglu, and G. Gulsen, "Simultaneous in vivo dynamic magnetic resonance-diffuse optical tomography for small animal imaging," J. Biomed. Opt. 13, 060501 (2008).

6. Z. Jiang, D. Piao, G. Xu, J. W. Ritchey, G. R. Holyoak, K. E. Bartels, C. F. Bunting, G. Slobodov, and J. S. Krasinski, "Trans-rectal ultrasound-coupled near-infrared optical tomography of the prostate, Part II: experimental demonstration," Opt. Express 16, 17505-17520 (2008).

7. B. Rivera, K. Ahrar, M. M. Kangasniemi, J. D. Hazle, and R. E. Price, "Canine transmissible venereal tumor: a large-animal transplantable tumor model," Comparative Med. 55(4), 335-343 (2005).

8. S. A. Bigler, R. E. Deering, and M. K. Brawer, "Comparison of microscopic vascularity in benign and malignant prostate tissue," Hum. Pathol. 24, 220-226 (1993). 